\title{
Research on product innovation design methods
}

\author{
WeiguoZhang ${ }^{1}$, JianyaoShu $^{2}, \mathrm{XitongHu}^{3}$ and YunMei $^{{ }^{*}}$ \\ ${ }^{1}$ Industrial design, University of Science and Technology Liaoning, Anshan City, Liaoning Province, 114051, China
}

\begin{abstract}
When the prehistoric man began to pick up stones, wood and other materials to make tools, we can confirm that creativity is not only the designer's personal patent but a natural gift of humanity. As product designers, we must have more creativity than ordinary people. By analyzing a large number of actual design works and award-winning works in international competitions for many years, the author summarizes the similarities of creative design and explores the application of innovative product design methods in design.
\end{abstract}

\section{Browse a large number of creative designs through design competitions}

Most industrial design competitions today are divided into product groups and concept groups. The product group is for products that are already on the market. The concept group is for creative ideas that don't need to be marketed. The focus is on the design creativity and problem solving methods of the contestants or some positive thinking about life. Participating in design competitions is a way to quickly verify that your creative ideas are reasonable and feasible, since the design competition is held every year, the design theme will be in line with future development trends. Therefore, the innovation and forward-looking of the award-winning works will inspire personal design thinking and design concepts. Therefore, you should browse the winning entries of the design competition and participate in the design competition actively. There are several internationally influential industrial design competitions: Red Star Award, Red Dot Award, IF Award, IDEA Award, Spark Award, A-Design, K-Design, Golden Dot Award, etc.

\section{Application of Innovative Design Method in Product Design}

\subsection{Product feature replacement}

A revolutionary innovation is likely to borrow from a technology in another area. When James Dyson used a vacuum cleaner to clean up his studio in 1978, he was troubled by the weakening suction of the vacuum cleaner, after disassembling the vacuum cleaner, he found that the dust collection bag inside the vacuum cleaner would be slowly blocked by dust, which caused the suction power to decrease. At the time he wanted to improve the problem without any clue. One day when he passed by the factory, he was inspired by the rapid separation effect of the cyclone device for separating paint particles by centrifugal force. James Dyson successfully invented the world's first bagless vacuum cleaner using the principle of centrifugal separation cyclone device. Based on Dyson's story, it leads to the first product creative design method, product feature replacement method.

Each product has its own product features, we understand how products are used by observing product features, and form a stronger directional thinking and usage habits. The design thinking of product feature replacement requires us to break the restricted product features and achieve improved product design by replacing other suitable product functional features. Use this design thinking to analyze a Red Dot award-winning work: 'figure 1'press jam. It uses the squeeze form feature of toothpaste and the take out form feature of canned jam to replace it. One of the product features of toothpaste is that it can be applied directly by squeezing a hose. At this time we need to find another product that can use the characteristics of toothpaste and bring a more friendly experience to the product. We found that using canned jam requires the following operations: open the bottle cap, dig out the jam with a spoon, and evenly spread the jam on the toast. If the way of using toothpaste is replaced with the way of using jam, it will greatly simplify the steps required for eating jam, and at the same time, it will bring a better user experience and improve hygiene issues for users.

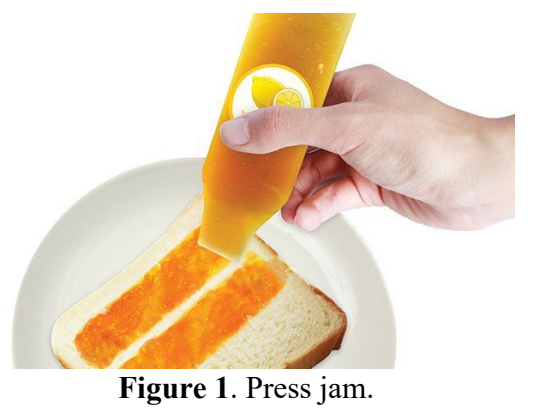




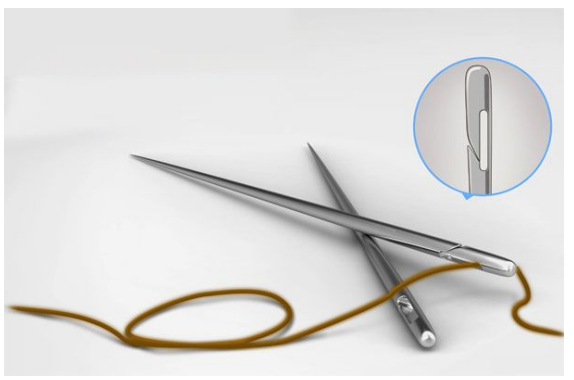

Figure 2. Clip pin.

Therefore, we can understand that the products in life can be extracted from certain characteristics and applied to another product, which has changed the user's use and operation to achieve an innovative design that optimizes the product experience. Designers need to take the initiative to find suitable functional features, and perform an innovative design with accurate replacement. How to accurately find these product characteristics is the key for designers in this innovative design method. Donald Norman proposes that everyone will be exposed to at least 20, 000 products in their lifetime. Designers should have the habit of refining product features, and 20,000 products will generate a huge knowledge base. Functional feature extraction and accumulation through products in daily life is enough to build a huge element library for us to design. In the text as 'figure 2', we can clearly see the application of product feature replacement thinking in design.so the inability to easily thread through the eyelets is a pain point. So finding a better connection that can replace the pin eye is the key to completing this innovative design. Finally, the designer extracted the functional features of the buckle that can connect two products and replaced the needle eye. Solved the pain point that the thread cannot be easily passed through the eye of the needle. It reduces the difficulty of product operation and enhances the product experience. In the text as 'figure 3', this is an awardwinning work of the LEXUS design award. The designer replaced the butter packaging lid, replacing the cover that only had a sealing function with a spatula, while satisfying the sealing function, it also adds the ability to directly apply butter. Eliminate the unnecessary consumption of other auxiliary tableware.

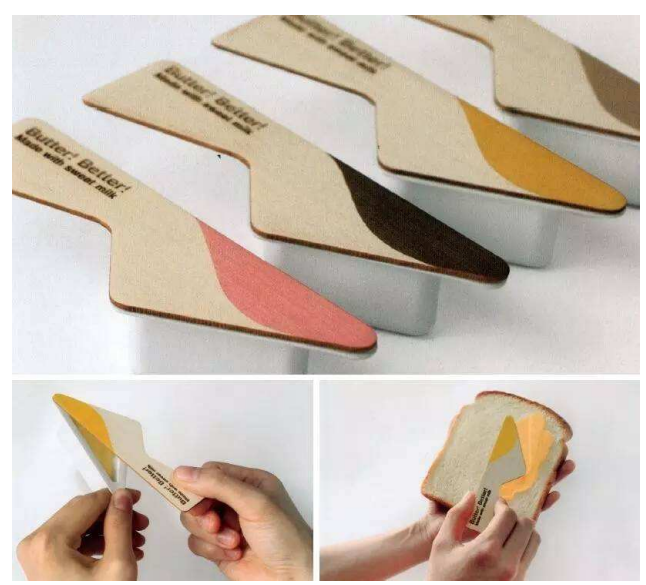

Figure 3. Butter box with knife Better! Better.
Many design cases have a common point to directly or indirectly replace product functions. Therefore, the author believes that reasonable feature replacement is one of the innovative product design methods.

\subsection{Related product function combination method based on scenario}

Observe that many of the products in our lives today are multifunctional products. 10 years ago, the juicer had only the function of juice extraction, and most of the current juicers today have functions such as juice extraction, boiled soybean milk, porridge cooking, etc. It can be found that these product functions are related, as if these functions were born together. Just like a printer is born with the functions of printing, copying and scanning. Compared with single-function products, multi-function products can better meet user needs and have more commercial value. The core of this method is to find the product creative design by finding the combination of related products in a common scenario. The combination of functions does not mean that the product functions are randomly combined. Only the effect of $1+1>2$ can meet the purpose of our product creative design. Refuse to over-design and avoid $1+1 \leq 2$ designs, which will lead to the decline of product usability. Designers should start with products that appear in the same scene or similar scenes in life, and look for opportunities that can be organically combined to form a creative design of $1+$ $1>2$.Let us analyze through design cases.

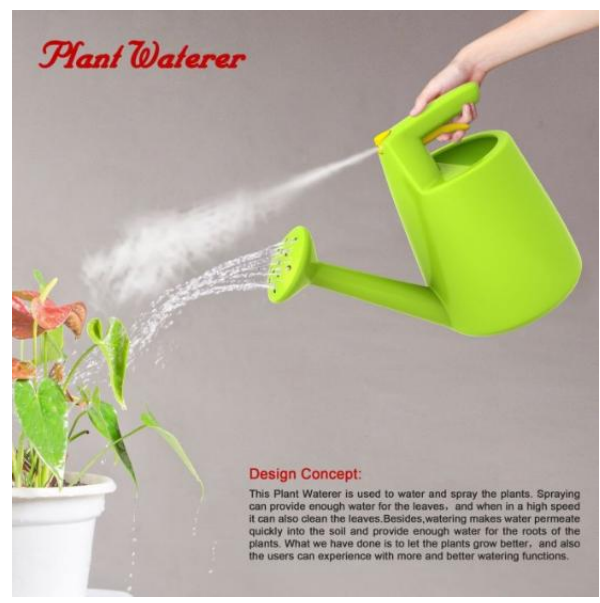

Figure 4. Multi-functional Sprinkler.

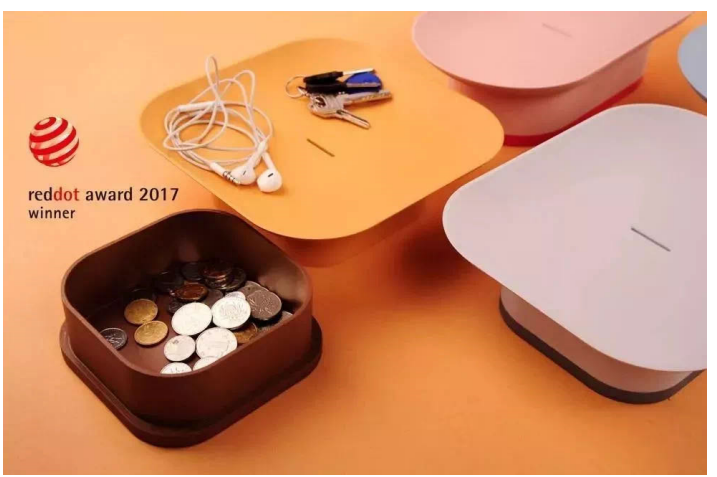

Figure 5. Tray piggy bank. 
As 'figure 4', Multi-functional Sprinkler is a typical combination of functions product, which achieves the effect of $1+1>2$. Watering flowers and spraying water are two actions based on the scene of growing flowers. Watering and water spraying, the frequency of crossover between the two is extremely high, so the designer combines the two products to improve the user experience in the watering process. 'Figure 5', the tray piggy bank is also based on this idea, combining the plate that holds small objects with the piggy bank, and the scenes of them are both on the desktop, so it can be naturally combined. Combined with the previously mentioned knowledge base, there are a lot of opportunities for single-function products in life that can be combined.

\subsection{Product function combination method based on scene continuity}

As mentioned above, the core of the product function combination method is to find products that can be combined based on the same or similar scenarios. For the user, the scene is dynamic rather than static, so the designer should also consider the products that can be designed due to the continuity of the scene. Therefore, combining products based on the continuity scene is also one of the important methods for creative design.

Because the three actions of washing vegetables, cutting vegetables, and frying vegetables have a relatively constant scene continuity. Therefore, the designer combines the product functions that meet the user behavior in these three scenarios, improves the cutting board, combines the three functions on the cutting board, and makes a cutting board meet the continuous use of the three scenarios of washing, cutting, and cooking. Thus completing the innovative design of the chopping board. As the 'Figure 6' shows. The design of 'Figure 7' also uses the same design method

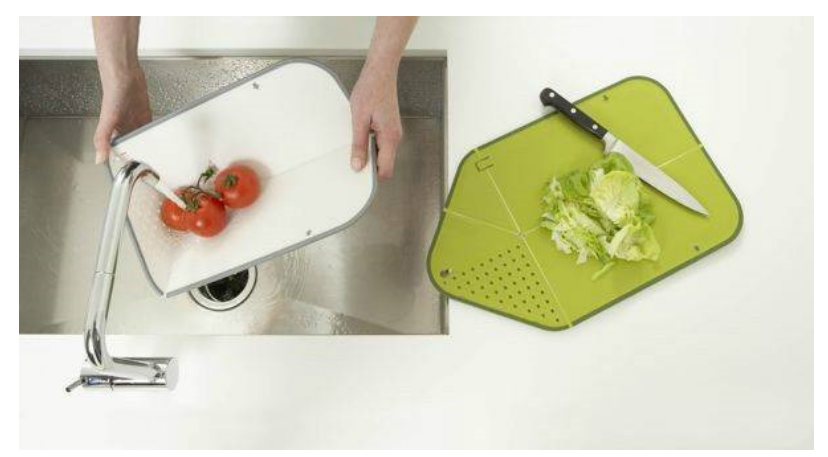

Figure 6. Foldable filter cutting board.

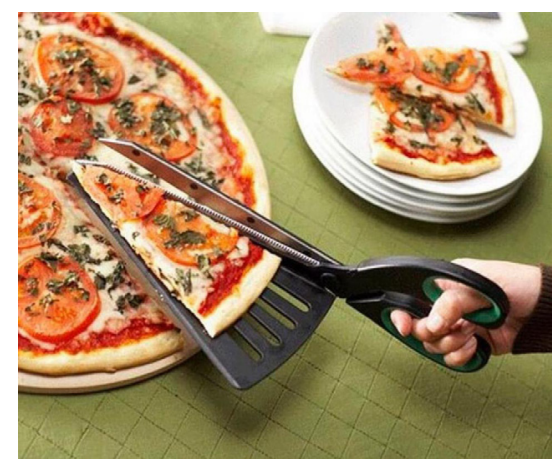

Figure 7. Pizza scissors shovel.

\subsection{Extract creative idea and redesign}

Newton once said: "If I look farther than others, it is because I am standing on the shoulders of giants." Redesigning by extracting good ideas from existing designs will undoubtedly make us become the man who standing on the shoulders of giants. Let's use the case for a more intuitive analysis.

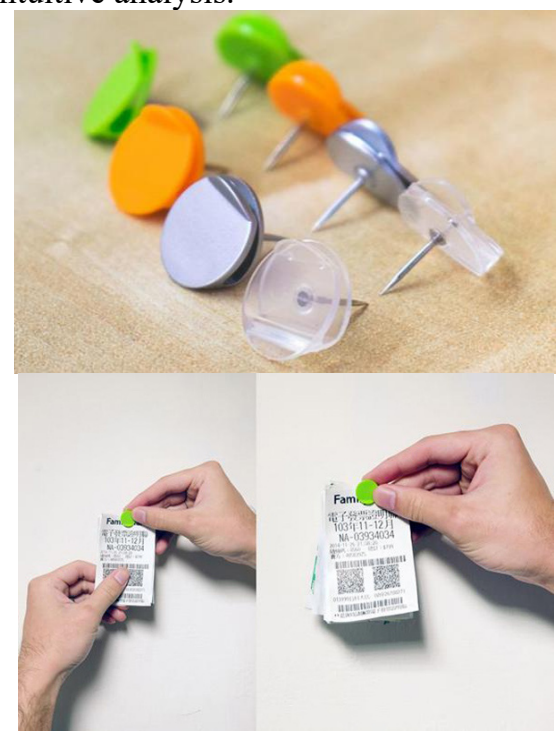

Figure 8. Easy Pin.
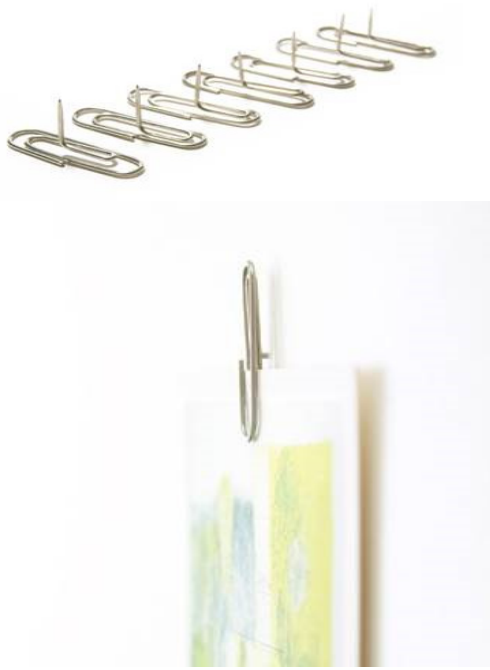

Figure 9. Pinclip Push Pin. 
Through the 'Figure 8'Easy Pin case, we can see that the designer used the design thinking of the related product function combination mentioned above, and combined the functions of the clip and the pushpin to solve the problem of breaking the paper when using the pushpin to fix the paper. The design also solves the problem that the pushpin is easy to roll and difficult to remove after use. Later, products similar to Easy Pin appeared, After that, as 'Figure 9', Pinclip Push Pin, a product similar to Easy Pin, appeared. By comparison, the design of the two pushpins has similar design points. Both have design points that do not damage the paper. Pinclip Push Pin uses a clip to replace the Easy Pin clip, which simplifies the processing process and the consumption of materials, and reduces the production cost of the product. The designer adopted a method of extracting creative idea and redesign. The method is not limited to the redesign of products in the same category. Take a 2010 Red Dot Award-winning, Accdion Package Folding Instant Noodle Bowl as an example, creative points that use the folding principle to save space are used in various types of product design. Until today's international competition, we will still see a variety of conceptual designs using the folding principle. Such as folding bathtubs, folding helmets, folding packaging, folding lighting, folding barricades, folding swimming circles, etc.

\section{Conclusion}

Borrow a word from the latest definition of industrial design by the World Design Organization (WDO) in 2015, "Industrial design is a strategic problem-solving process that drives innovation through innovative products, systems, services, and experience, builds business success, and leads to a better quality of life." Design is already in the 3.0 era, and the people-oriented design concept must always be embedded in our innovative design. Innovative design methods are not formulas, but the process of design realization. This article lists several product creative design methods and their application cases in design. In practical applications, designers need to flexibly apply innovative design methods according to actual requirements.

\section{References}

1. Liang, Y. (2019) Research on the Application of Innovative Design Method in Product Design. Industrial design., 10: 138-139.

2. Zhang, J. (2017) Conceptual Productization and Innovative Design Methods_-Driving Design Teaching with International Design Competition. Decoration., 10: 108-110.

3. Wang, D. (2018) Research on Cultivation of Creative Thinking in Product Design Teaching-_ From Problem Discovery to Design Verification. Industrial design., 02: 98-99.

4. Dai, S.T. (2016) Solving problems with design. IBeijing Times Chinese Press, Beijing.
5. Zhang, Y.S., (2019) Producct Thinking:6 innovative principles of design. Publishing House of Eletronics Industry, Beijing. 